\title{
Estimating along-track plasma drift speed from electron density measurements by the three Swarm satellites
}

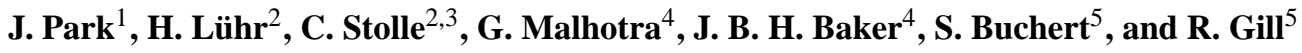 \\ ${ }^{1}$ Korea Astronomy and Space Science Institute, Daejeon, Korea \\ ${ }^{2}$ GFZ, German Research Center for Geosciences, Potsdam, Germany \\ ${ }^{3}$ Faculty of Science, University of Potsdam, Potsdam, Germany \\ ${ }^{4}$ Bradley Department of Electrical and Computer Engineering, Virginia Tech, Blacksburg VA, USA \\ ${ }^{5}$ Swedish Institute of Space Physics, Uppsala, Sweden \\ Correspondence to: J. Park (pj@kasi.re.kr)
}

Received: 26 December 2014 - Revised: 28 May 2015 - Accepted: 15 June 2015 - Published: 08 July 2015

\begin{abstract}
Plasma convection in the high-latitude ionosphere provides important information about magnetosphereionosphere-thermosphere coupling. In this study we estimate the along-track component of plasma convection within and around the polar cap, using electron density profiles measured by the three Swarm satellites. The velocity values estimated from the two different satellite pairs agree with each other. In both hemispheres the estimated velocity is generally anti-sunward, especially for higher speeds. The obtained velocity is in qualitative agreement with Super Dual Auroral Radar Network data. Our method can supplement currently available instruments for ionospheric plasma velocity measurements, especially in cases where these traditional instruments suffer from their inherent limitations. Also, the method can be generalized to other satellite constellations carrying electron density probes.
\end{abstract}

Keywords. Ionosphere (Plasma convection)

\section{Introduction}

Plasma convection in the high-latitude ionosphere is one of the key pieces of information for the magnetosphereionosphere-thermosphere coupling. The convection has an influence on plasma irregularity generation and transport (e.g. Zhang et al., 2013), the fine structure of the polar cap aurora (e.g. Sojka et al., 1994), energy deposition into the thermosphere (e.g. Matsuo and Richmond, 2008), and even the initial evolution of substorms (e.g. Nishimura et al., 2014).
Hence, plasma drift measurements in the high-latitude ionosphere are of considerable importance.

Plasma convection can be measured in several different ways. The Super Dual Auroral Radar Network (SuperDARN) consists of dense networks of ground-based radars, which measure line-of-sight (LOS) drift speed of decametrescale ionospheric irregularities. By combining drift measurements from several radars, maps of plasma velocity can be derived. The SuperDARN global convection maps typically depict data captured every 1-2 min. The Defense Meteorological Satellite Program (DMSP) satellites also measure ion drift velocities regularly. The SuperDARN and DMSP observations have been conducted nearly continuously and on a regular basis. Occasionally, ground-based optical instruments (e.g. Hosokawa et al., 2006) and Global Positioning System (GPS) receivers (e.g. Benton and Mitchell, 2012) can track ionospheric irregularities to estimate plasma drift velocity.

Although high-latitude plasma convection has been monitored with a number of methods, each has its own limitations. SuperDARN radars cannot measure the convection when there are no decametre-scale irregularities in the ionosphere, which limits its performance during extremely low and high geomagnetic activity. The DMSP measurements are constrained by the satellite orbits, which have poor spatial coverage in the post-midnight and post-noon local time (LT) sector. As for the temporal resolution, the convection data for the polar region can be given only every $\sim 100 \mathrm{~min}$, which is the orbit period of the DMSP satellites. Furthermore, the along-track component of the convection velocity 
measurements is hardly used because of poorer quality compared with the cross-track components. Ground-based optical and/or GPS observations can be a good supplement to SuperDARN and DMSP observations, but the data coverage is usually limited to continental areas where the instruments are located. In particular, the operation of optical measurements is normally restricted to dark periods when the Sun is below the horizon. Therefore, more independent measurements of high-latitude plasma convection velocity are still warranted.

In this study we introduce an automatic method to estimate plasma drift velocity in the high-latitude ionosphere using the Swarm constellation. Though a similar method was used in case studies of Spicher et al. (2015) and Goodwin et al. (2015), our method is fully automatic and needs no human intervention. The three Swarm satellites carry instruments that directly measure ion drift velocity (thermal ion imager in the electric field instrument suite). However, this paper discusses another method for along-track plasma velocity estimation, which solely uses electron density profiles measured by the onboard Langmuir Probe (LP). In Sect. 2 we will briefly describe the instruments and analysis methods. The statistical results will be shown and discussed in Sect. 3 . Finally, we summarize our results and draw conclusions in Sect. 4.

\section{Instruments and data processing}

The Swarm constellation consists of three identical satellites, which were launched on 22 November 2013 into a nearcircular polar orbit (orbit inclination angle $\sim 87.5^{\circ}$ ) (FriisChristensen et al., 2008). The initial altitudes of the three satellites were nearly the same $(\sim 500 \mathrm{~km})$ during the commissioning phase until mid-January 2014, after which satellite orbits gradually separated regarding altitudes and longitudes. Not all the instruments were in full operation during the commissioning phase. Here we use only the initial electron density data measured by the Swarm LP. Further, we consider the data between 09 December 2013 and 15 January 2014 (hereafter, "the mission period of interest"), when the three satellites were at similar altitudes and longitudes.

During the mission period of interest the ascending nodes of the Swarm orbits were generally located between 10:00 and 14:00 LT, i.e. the satellite tracks were close to the noonmidnight meridian. The zonal separation of adjacent orbital planes was less than $1^{\circ}$ near the equator (i.e. $<110 \mathrm{~km}$ ) (e.g. Lühr et al., 2015; Fig. 1), which decreases further at high latitudes. The latitudinal separation of the three satellites was non-negligible. Swarm-B was the leading satellite, while Swarm-A (later by $<\sim 1 \mathrm{~min}$ ) and Swarm-C (later by $<\sim 3$ min than Swarm-B) followed in that order. Under this formation the three satellites were nearly aligned along one orbit track at high latitudes, like pearls on a string. They could encounter similar plasma density structures one satellite after another.

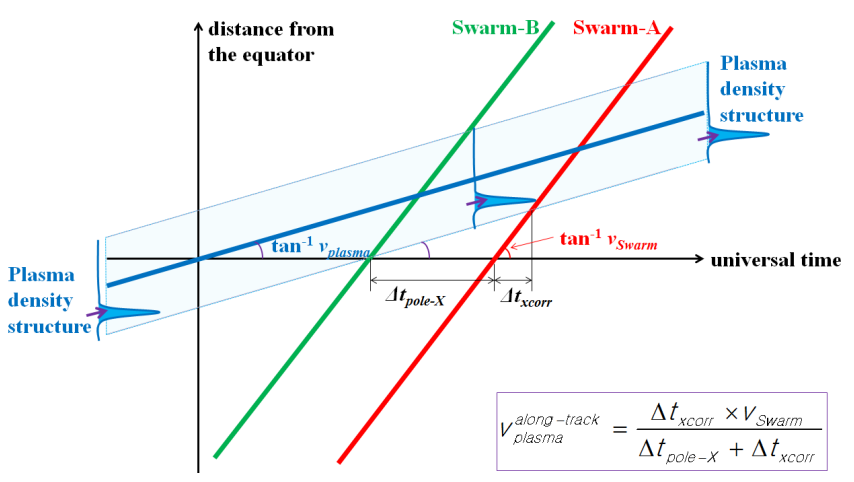

Figure 1. A schematic diagram showing the relative motion of the Swarm satellites and plasma density structures.

Figure 1 shows our strategy to estimate along-track plasma drift speed using a pair of the Swarm satellites. The horizontal axis represents Universal Time (UT), and the vertical axis represents the distance from the equator. The red and green lines are the trajectories of Swarm-A and Swarm-B, respectively. Both satellites are assumed to move with the same speed, $v_{\text {Swarm. }}$. The blue area signifies the path of a plasma density structure. Its speed along the Swarm orbit $\left(v_{\text {plasma }}^{\text {along-track }}\right)$ and the morphological shape are assumed to be time-independent during the passages of the Swarm satellites (a few minutes). As mentioned previously, Swarm-A is behind Swarm-B by about $<\sim 1 \mathrm{~min}$ : this time difference is denoted as $\Delta t_{\text {pole }-X}$, which means pole-crossing time difference. The difference between the times when Swarm-B and Swarm-A encounter the plasma density structure is not $\Delta t_{\text {pole }-X}$. Since the plasma density structure is also moving, there appears an additional time difference, $\Delta t_{x \text { corr }}$. This additional time difference can be estimated by cross-correlating the two density profiles measured by Swarm-B and SwarmA. Applying simple trigonometric equations to Fig. 1, the plasma drift speed along the Swarm track ( $\left.v_{\text {plasma }}^{\text {along-track }}\right)$ can be estimated from the known values of $v_{\text {Swarm }}\left(\sim 7.5 \mathrm{~km} \mathrm{~s}^{-1}\right)$, $\Delta t_{\text {pole- } X}$ (obtained from the Swarm ephemeris data) and $\Delta t_{x \text { corr }}$ (obtained from the Swarm LP data cross-correlation):

$v_{\text {plasma }}^{\text {along-track }}=\frac{\Delta t_{x \text { corr }} \times v_{\text {Swarm }}}{\Delta t_{\text {pole }-X}+\Delta t_{x \text { corr }}}$.

Note that the plasma density profiles measured by the Swarm satellites differ from the "true snapshots" of the density structure. True snapshots can be obtained only when $v_{\text {Swarm }}$ becomes infinite (i.e. when the green and red lines in Fig. 1 become vertical). The only two assumptions used to derive Eq. (1) are the time independence of (a) $v_{\text {plasma }}^{\text {along }}$ and (b) the morphology of the plasma density structure. These assumptions can be justified by the fact that $v_{\text {Swarm }}$ (about $7500 \mathrm{~m} \mathrm{~s}^{-1}$ ) is usually much faster than $v_{\text {plasma }}^{\text {along-track }}$ (of the order of $100 \mathrm{~m} \mathrm{~s}^{-1}$ ). Note also that $v_{\text {plasma }}^{\text {along-track }}$ is the speed with respect to a ground observer. A plasma density irregu- 
larity fixed in geographic coordinates (e.g. above Greenland) would be encountered by different Swarm satellites at the same location. As a result, $\Delta t_{x \text { corr }}$ becomes 0 , which yields a zero value for $v_{\text {plasma }}^{\text {along }}$.

In applying this method to Swarm LP data, we use the leading satellite, Swarm-B as the reference satellite. We first divide the Swarm-B (leading satellite) data into $1 \mathrm{~min}$ segments, which are advanced in steps of $20 \mathrm{~s}$ through the continuous Swarm-B data stream. For example, the first segment of the Swarm-B data lasts from 00:00:00 to 00:01:00 UT, and the next data interval from 00:00:20 to 00:01:20 UT. Segments containing a single data gap longer than $1 \mathrm{~s}$ or cumulated gaps longer than or equal to $20 \%$ of the expected segment length are neglected. In both hemispheres only data within $\pm 5 \min (\sim \pm 2300 \mathrm{~km})$ around the peaks of absolute geographic latitude (GLAT) are considered because the intersatellite zonal distance becomes larger at lower latitudes. As a next step we also divide the Swarm-A and Swarm-C data into 1 min data segments, taking into account the time difference of the pole crossing $\left(\Delta t_{\text {pole }-X}\right)$ with respect to SwarmB (see also Ritter et al., 2013). In this way all the 1 min data segments from the three satellites correspond to nearly the same location. Note that $\left|\Delta t_{\text {pole }-X}\right|$ should be $\leq 40 \mathrm{~min}$ in order for the segments to be analysed further.

Figure 2 shows one example of plasma density profiles processed in the above-mentioned way. In this figure different colours are used for the satellites: red for Swarm-A, green for Swarm-B, and blue for Swarm-C. The satellite tracks are shown in geographic coordinates in panel (a) while panel (b) presents those in apex magnetic latitude and magnetic local time (MLT) coordinates. Small circles in Fig. 2a-b correspond to the time stamps given in panels (c)-(e) while the triangle denotes the start of the time series. There are actually three individual satellite tracks in panels (a)-(b). However, the three satellite tracks are hardly distinguishable, consistent with the assumption that the data segments shown in panels (c)-(e) correspond approximately to the same location. Panel (b) shows that the three satellites were on the prenoon side and poleward of $80^{\circ}$ in magnetic latitude (MLAT).

Panels (c)-(e) present electron density measured by the Swarm LP. Note the different time stamps of the three panels. The satellite altitudes given in panels (c)-(e) agree with one another within $1 \mathrm{~km}$. Swarm-B (panel d, green) first passed the polar region and encountered plasma density structures. About 1 min after Swarm-B, Swarm-A (panel c, red) passed the same region. About 2 min after Swarm-B, Swarm-C (panel e, blue) passed the same region. The three density profiles appear strikingly similar, only displaced along the $x$ axis.

The green lines overplotted in panels (c) and (e) represent the density profile measured by Swarm-B (panel d, green) but time-shifted by $\Delta t_{\text {pole }-X}$ : this is the plasma density profile the trailing Swarm satellites (Swarm-A or Swarm-C) would encounter if the along-track plasma drift speed was (a)

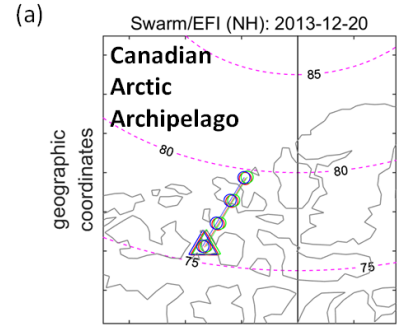

(b)

(c)

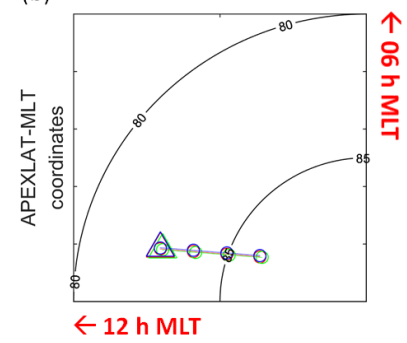

(d)
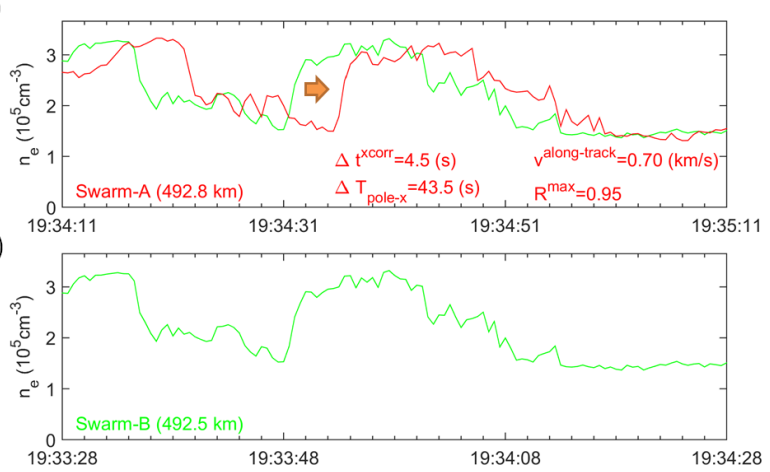

(e)

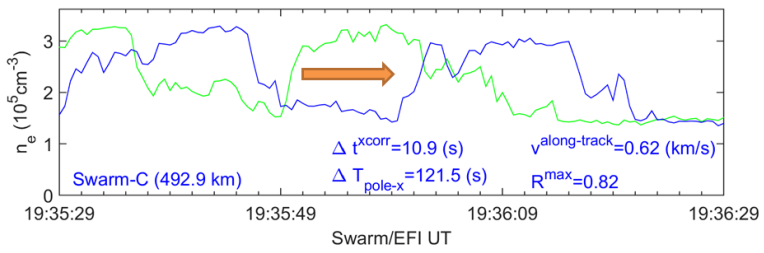

Figure 2. An example of Swarm LP data: (a) satellite track in geographic coordinates, (b) satellite track in apex magnetic latitude and MLT coordinates, (c-e) electron density measured by the LP onboard Swarm-A (red), Swarm-B (green), and Swarm-C (blue). Circles in panels (a)-(b) correspond to the time stamps of panels (c)-(e), and triangles mark the start of the time series. The green lines overplotted in panels (c) and (e) represent the density profile measured by Swarm-B but time-shifted by $\Delta t_{\text {pole }-X}$.

$0\left(v_{\text {plasma }}^{\text {along-track }}=0\right)$. We cross-correlate this profile (timeshifted Swarm-B profile) with those of the respective trailing satellites (Swarm-A or Swarm-C). All profiles are linearly detrended and interpolated in an automatic way prior to cross-correlation. For both of the Swarm-B-A and SwarmB-C pairs, cross-correlation between the density profiles leads to maximum correlation coefficients $\left(R^{\max }\right)$ higher than 0.8 . The time shift corresponding to the maximum correlation coefficient is used to estimate the additional time difference $\left(\Delta t_{x \text { corr }}\right)$ in Fig. 1 . Then the along-track velocity of the plasma density profile $\left(v v_{\text {plasma }}^{\text {along-track }}\right)$ is obtained from Eq. (1). Each satellite pair (i.e. Swarm-B-A and Swarm-B-C) leads to one value of along-track velocity of the plasma density profile $\left(v_{\text {plasma }}^{\text {along-track }}\right)$. In Fig. 2 the calculation results of 700 (620) $\mathrm{m} \mathrm{s}^{-1}$ are listed for the Swarm-B-A (for Swarm-B-C) pairs, which agree with each other to within $13 \%$. Note that a positive velocity corresponds to plasma drift in the flight di- 
rection of the Swarm satellites (negative velocity corresponds to plasma drift ramming into Swarm). Hence, the along-track velocity of the plasma density profile is nearly anti-sunward in the example shown in Fig. 2.

\section{Statistical results and discussion}

\subsection{Velocity estimation using Swarm}

We applied the method described in Sect. 2 to all the Swarm observations between 09 December 2013 and 15 January 2014. To check the robustness and consistency of velocity estimates, only those days are used when all the three satellites observed electron density, i.e. a day is neglected when one of the satellites did not operate the onboard LP. Further, we only consider the polar passes with significant fluctuations in plasma density. If no substructures are observed, the density profiles seen by the three Swarm satellites are likely to be similar, with a high maximum correlation coefficient for $\Delta t_{x c o r r}=0$. This would indicate a zero along-track velocity even though the plasma is moving. We remove the slowly varying background from the plasma density profile by means of a Savitzky-Golay filter (order: 2; window size: 31 data points). Considering the filter cutoff period, the residual density is constrained to fluctuations of less than about $80 \mathrm{~km}$ scales. If the mean absolute value of the residual is smaller than $1.2 \times 10^{4} \mathrm{~cm}^{-3}$, the orbit segment is omitted in further data processing.

To avoid false velocity estimation, we impose a rather strict condition for the morphological similarity between plasma density profile pairs: the maximum cross-correlation between plasma density profiles should be higher than 0.65 . Figure 3 shows the correlation diagram of the along-track plasma drift speed obtained by the Swarm-B-A pair and the Swarm-B-C pair. Each data point in Fig. 3 corresponds to one 1 min data segment, as shown in Fig. 2. Blue and red crosses represent observations in the Northern and Southern Hemisphere, respectively. Note that speed estimates exceeding $1 \mathrm{~km} \mathrm{~s}^{-1}$ are omitted from the figure. Figure 3 shows that the two independent speed estimates from the two satellite pairs exhibit high correlation $(\sim 0.9)$, and most values are concentrated around the line of perfect correspondence (unity slope). This good agreement suggests that the method for plasma velocity estimation (Eq. 1) is reliable and robust except for a few outliers.

Note that the leading satellite, Swarm-B was used as the reference satellite in producing Fig. 3. We have repeated the same procedure while changing the reference satellite to Swarm-A and Swarm-C. All the results (figures not shown) look qualitatively similar to Fig. 3, with the correlation coefficient between the abscissa and the ordinate being 0.83 (0.96) when Swarm-A (Swarm-C) is used as the reference satellite.

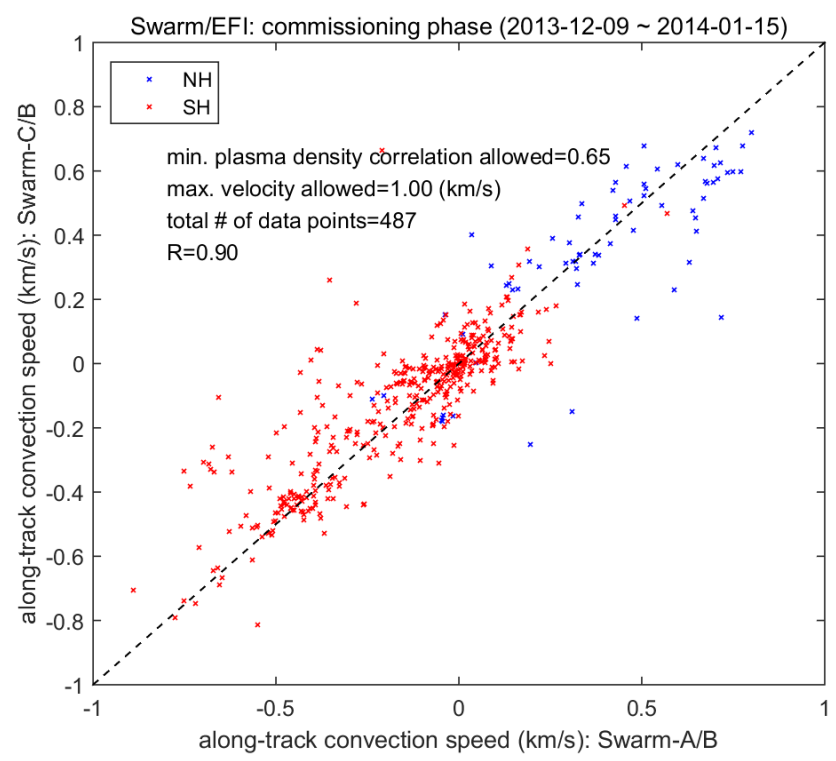

Figure 3. Correlation diagram between the along-track plasma drift speed obtained by the Swarm-B-A pair and the Swarm-B-C pair. Each data point corresponds to one 1 min data segment, as shown in Fig. 2. Blue and red crosses represent observations in the Northern and Southern Hemisphere, respectively. The dashed line represents perfect correspondence between the abscissa and the ordinate.

Goodwin et al. (2015) conducted similar analyses using Swarm data (for a few cases of polar cap patches). In Table 1 of Goodwin et al. (2015) the along-track plasma drift speed is (1) mostly below $500 \mathrm{~m} \mathrm{~s}^{-1}$, with a mean of about $280 \mathrm{~m} \mathrm{~s}^{-1}$, and (2) occasionally below $100 \mathrm{~m} \mathrm{~s}^{-1}$. In Fig. 3 we can see that the range of -500 to $+500 \mathrm{~m} \mathrm{~s}^{-1}$ contains the majority of the population. Hence, our Fig. 3 generally agrees with Goodwin et al. (2015); this also supports the validity of our method.

In Fig. 3 we can see that the along-track plasma convection speed is mostly positive (negative) in the Northern (Southern) Hemisphere. During the mission period of interest, the Swarm orbital directions point approximately from noon to midnight in the Northern Hemisphere (NH) and reversed in the Southern Hemisphere (SH). As positive velocity corresponds to plasma drift in the flight direction of the Swarm satellites (see Sect. 2), Fig. 3 shows that the plasma drift is generally from noon to midnight (i.e. anti-sunward) in both hemispheres, especially for high speeds beyond about $\pm 300 \mathrm{~m} \mathrm{~s}^{-1}$.

The numbers of data points in Fig. 3 are 70 and 417 for the $\mathrm{NH}$ and $\mathrm{SH}$, respectively. This ratio of 1 to $6(=0.17)$ in favour of the Southern Hemisphere can be explained in the following way. First, we should consider sampling bias. The total number of paired (e.g. between Swarm-B and SwarmA) 1 min data segments analysed, as described in Fig. 2, is 39353 and 39485 for the $\mathrm{NH}$ and $\mathrm{SH}$, respectively: the ratio between the former and the latter is about unity. Second, 

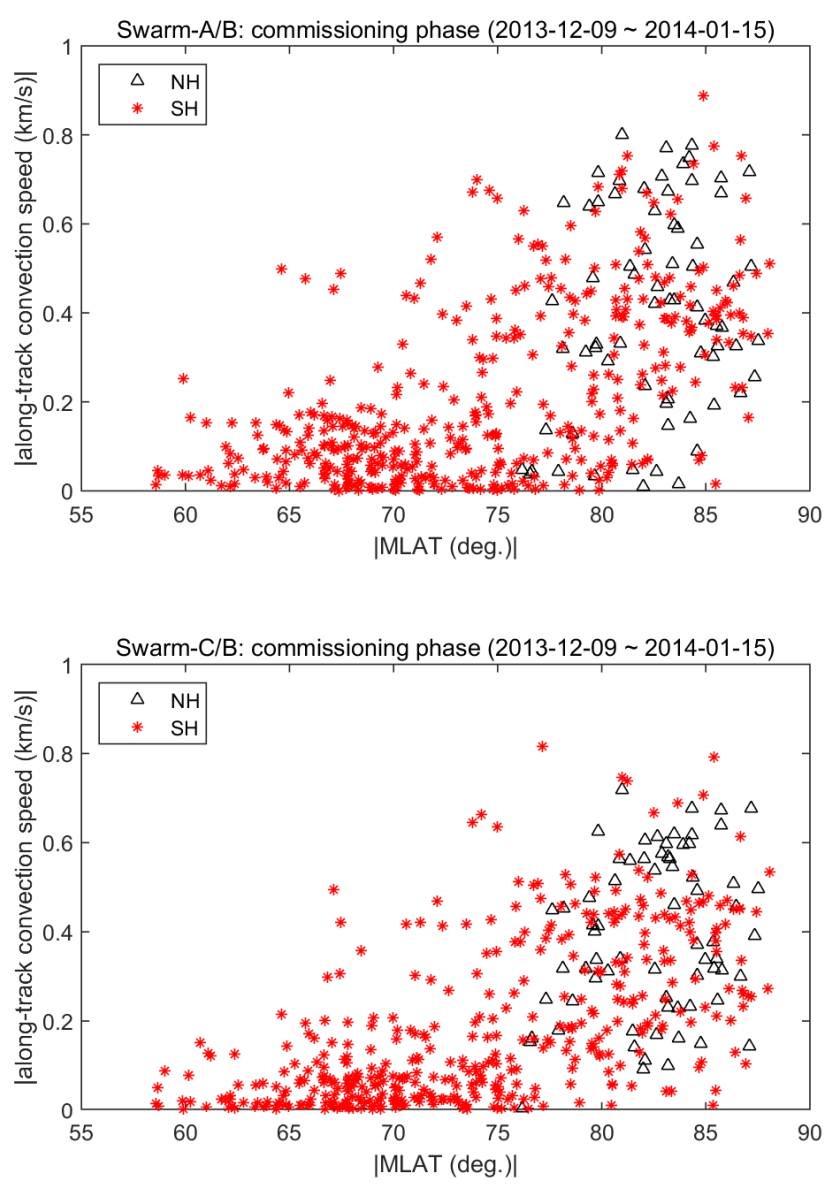

Figure 4. $\mid$ MLAT $\mid$ dependence of the along-track plasma drift speed obtained by (a) the Swarm-B-A pair and (b) by the Swarm-B-C pair.

Noja et al. (2013) found that $40 \%(60 \%)$ of plasma density irregularities observed at $|\mathrm{MLAT}| \geq 55^{\circ}$ by the CHAMP (Challenging Minisatellite Payload) satellite belong to the $\mathrm{NH}(\mathrm{SH})$. The irregularities' natural preference for the $\mathrm{SH}$ can contribute to the hemispheric asymmetry in our Fig. 3. The sampling bias (about unity) in combination with irregularities' natural preference for the $\mathrm{SH}(0.67)$ would result in a north-to-south ratio of $0.67(=1 \times 0.67)$, which is still insufficient to explain the ratio observed in Fig. 3 (0.17). Hemispheric asymmetry in distributions of the MLAT ranges analysed in this study, which will be discussed at the end of this subsection, can make an additional contribution. However, further study is needed to elucidate quantitatively the hemispheric asymmetry in the number of events shown in Fig. 3.

We have also investigated the dependence of $v_{\text {plasma }}^{\text {along-track }}$ on $\mid$ MLAT $\mid$ in Fig. 4. The top (bottom) panel presents $v_{\text {plasma }}^{\text {along-track }}$ obtained by the pair Swarm-A and Swarm-B (Swarm-C and Swarm-B). Black triangles and red asterisks correspond to the $\mathrm{NH}$ and $\mathrm{SH}$, respectively. As $|\mathrm{MLAT}|$ increases, the upper bound of the along-track plasma convection speed in- creases, while the lower bound stays more or less unchanged. That is to say, $v_{\text {plasma }}^{\text {along-track }}$ at lower $\mid$ MLAT $\mid$ (e.g. at auroral regions) is generally lower, while $v_{\text {plasma }}^{\text {along-track }}$ at high $\mid$ MLAT $\mid$ (e.g. inside the polar cap) can exhibit a wide range of values. This MLAT dependence can be explained in the following way. Unless the interplanetary magnetic field (IMF) is directed strongly northward, (1) the convection velocity for $|\mathrm{MLAT}| \geq 80^{\circ}$ (in the polar cap) on average has a dominant noon-to-midnight component but (2) convection velocities at $|\mathrm{MLAT}|<75^{\circ}$ (in the auroral region) generally exhibit reduced magnitude and/or deviations from the noon-tomidnight direction (Förster and Haaland, 2015; Figs. 2-3). When the IMF is directed strongly northward, the convection velocity shows complex patterns regardless of whether |MLAT| is above $80^{\circ}$ or below $75^{\circ}$ (Förster and Haaland, 2015; Figs. 2-3). As mentioned in the preceding paragraph, the Swarm orbital directions are approximately aligned with the noon-midnight direction during the mission period of interest. Hence, the along-track convection velocity component measured by Swarm is likely to be larger in magnitude within the polar cap than at auroral latitudes.

In Fig. 4 events in the $\mathrm{NH}$ are generally confined to the high-latitude region of $|\mathrm{MLAT}|>75^{\circ}$. On the other hand, a significant part (about $75 \%$ ) of the events in the SH comes from $\mid$ MLAT $\mid<75^{\circ}$. This hemispheric asymmetry is as expected from our data selection method described in Sect. 2. Only data within $\pm 5 \mathrm{~min}$ (approximately $\pm 2300 \mathrm{~km}$ or $\pm 20^{\circ}$ in latitude) around the peaks of $\mid$ GLAT $\mid$ are selected and analysed in this study. The offset between the geographic and geomagnetic poles is larger in the SH than in the NH. Hence, wider ranges of $|\mathrm{MLAT}|$ can be populated well by the selected and analysed Swarm data in the SH than in the NH.

\subsection{Swarm-SuperDARN comparison}

We further compared the data with independent ground observations conducted by SuperDARN. To ensure that the plasma drift speed measured by Swarm does not change within a few minutes (that is, to focus only on the bulk population around the $1: 1$ correspondence line in Fig. 3), Swarm $v_{\text {plasma }}^{\text {along-track }}$ data are used only when they satisfy the following conditions:

1. the mean of the two $v_{\text {plasma }}^{\text {along-track }}$ estimates is smaller in magnitude than $1000 \mathrm{~m} \mathrm{~s}^{-1}$;

2. the difference in $v_{\text {plasma }}^{\text {along-track }}$ for the Swarm-B-A pair and the Swarm-B-C pair is smaller than $40 \%$ of the mean of the two $v_{\text {plasma }}^{\text {along-track }}$ estimates (note that these two estimates are separated in time by a few minutes); and

3. the MLT should be within $\pm 3.5 \mathrm{~h}$ of noon or midnight. Plasma convection in the dawn-dusk sector may exhibit strong latitudinal shear, which may hinder the inter- 
instrument comparison due to the proximity to the convection reversal boundary.

Plasma velocities from the SuperDARN map velocity data (at 2 min cadence) are chosen for comparison when they satisfy the following conditions:

1. the centre point of each 1 min segment of Swarm-B data lies within $\pm 1^{\circ}$ in latitude and $\pm 10^{\circ}$ in longitude of the altitude adjustment corrected geomagnetic (AACGM) coordinates of the SuperDARN grid point, and within \pm 4 min in time and

2. more than one grid point of SuperDARN data satisfies Condition (1).

If satisfied, the SuperDARN map velocity data at those candidate grid points are

1. projected onto the horizontal velocity vector of Swarm$\mathrm{B}$ orbit $\left(v_{\mathrm{Swarm}}^{\text {north }}, v_{\mathrm{Swarm}}^{\text {east }}\right)$ for each candidate grid point (displacement of the actual Swarm-B location from the SuperDARN data point is neglected for simplicity);

2. averaged over different candidate grid points; and

3. compared with the along-track plasma speed estimation from Swarm electron density profiles $\left(v_{\text {plasma }}^{\text {along-track }}\right)$.

The results of this Swarm-SuperDARN comparison are shown in Fig. 5. The $x$ axis represents the mean of $v_{\text {plasma }}^{\text {along-track }}$ from the Swarm-A-B and B-C pairs, while the $y$ direction shows SuperDARN map velocities projected onto the Swarm flight direction. The correlation coefficient of the two plasma velocities estimated from Swarm and SuperDARN is about 0.75 , and the total number of common events is 34 . Note also that most of the data points in Fig. 5 are not too far from the line of perfect correspondence (i.e. the line of unity slope). Nevertheless, the slope of the best fit lines is only 0.54 when we use the simple linear regression methods. This indicates that we obtain clearly larger velocities from Swarm estimates than from SuperDARN reconstructions. If we assume comparable uncertainties in the two data sets (i.e. using the total linear regression method), the slope increases to 0.64 , which means the SuperDARN results are on average smaller by about $36 \%$. However, the bias between the two types of measurements (about $50 \mathrm{~m} \mathrm{~s}^{-1}$; see Fig. 5) is quite small (i.e. much smaller than the natural variation range in Fig. 5).

We have repeated the same procedure while changing the reference satellite from Swarm-B to Swarm-A and Swarm-C. All the results (figures not shown) look qualitatively similar to Fig. 5, with the correlation coefficient between the abscissa and the ordinate being 0.75 (0.67) when Swarm-A (SwarmC) is used as the reference satellite.

Reasons for the imperfect, though reasonable, agreement $(R \sim 0.75)$ between Swarm and SuperDARN estimates may

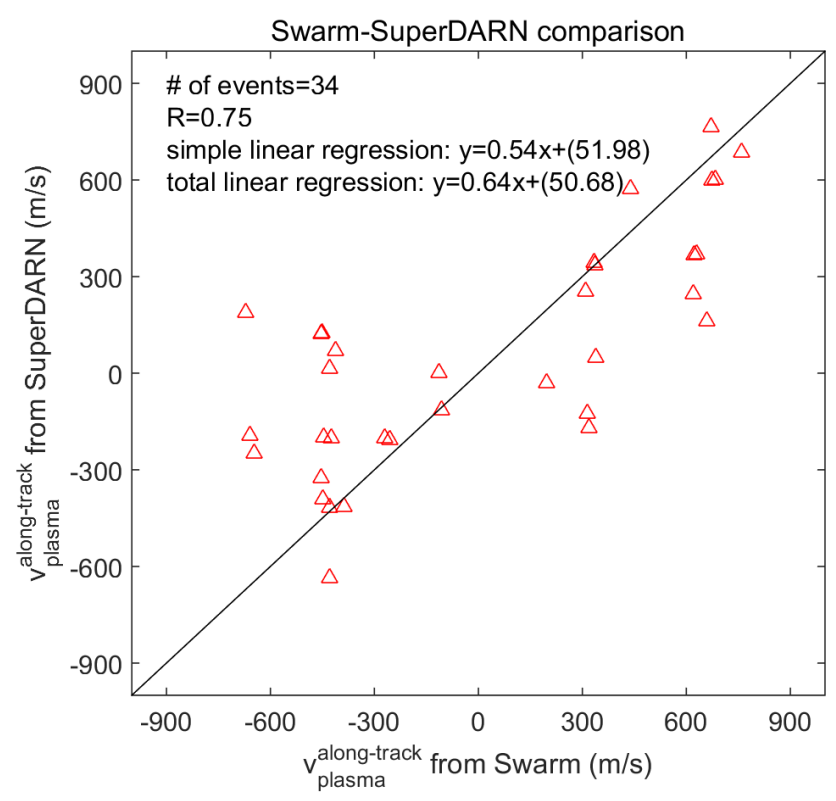

Figure 5. Correlation diagram for the along-track plasma drift speed obtained by Swarm and SuperDARN. The diagonal line represents perfect correspondence between the abscissa and ordinate.

be explained as follows. The SuperDARN map velocity data represent large-scale convection patterns because of their beam width and consequent grid spacing. This smoothing probably leads to smaller velocities. In contrast, Swarm satellites track individual patches at high latitudes. Hence, differences between large-scale convection patterns (SuperDARN) and smaller-scale plasma drift features (Swarm), especially around plasma density irregularities (e.g. Nishimura et al., 2014; Zou et al., 2015; Yang et al., 2015), are expected to result in a reduced correspondence between the two velocity estimates. Furthermore, Yang et al. (2015, Fig. 4) demonstrate that the SuperDARN velocity is on average $18 \%$ lower in magnitude than that estimated from the motion of pulsating auroral patches. These results can partly explain the systematic underestimation in our Fig. 5 (underestimation by $36 \%)$.

The multi-satellite method, which is based on a pearls-ona-string constellation, can be a useful method to determine plasma velocity along the string of satellites. After completion of the operational constellation (by mid-April 2014), the Swarm-A and Swarm-C satellites are again at the same altitudes with a small zonal separation $\left(\sim 1.4^{\circ}\right.$ around the equator). However, the temporal separation between the satellites is rather small $(\sim 9 \mathrm{~s})$ such that the presented method can hardly be applied in a relable manner. According to Eq. (1), for example, an along-track plasma speed of $0.5 \mathrm{~km} \mathrm{~s}^{-1}$ will result in a $0.64 \mathrm{~s}$ time shift $\left(\Delta t_{x \text { corr }}\right)$ between Swarm-A and Swarm-C observations. This time difference is close to the nominal sampling period $(0.5 \mathrm{~s})$ of the Swarm LP; therefore, 
improved methods are needed for the estimation of alongtrack plasma speed from these data.

\section{Summary and conclusion}

Using electron density measured by the Swarm constellation between 01 December 2013 and 15 January 2014, we have estimated the along-track velocity component of plasma convection within and around the polar cap. Our main conclusions can be summarized as follows:

1. The velocity values estimated from two different satellite pairs agree well with each other.

2. Further, the velocity exhibits reasonable agreement with plasma velocity from SuperDARN, at least for those cases which coincide with the Swarm observations. On average, SuperDARN data reveal drift velocities smaller by $36 \%$ than those from the local Swarm observations.

3. In both hemispheres the estimated velocity is more frequently anti-sunward rather than sunward, as expected.

Our method can supplement currently available instruments for ionospheric plasma velocity measurements (e.g. SuperDARN and DMSP satellites), especially in cases where these traditional instruments suffer from their inherent limitations (e.g. along-track component of ion drift metres). Also, the method can be generalized to other satellite constellations carrying similar electron density probes.

Acknowledgements. The authors thank the reviewer, who gave very constructive comments. The European Space Agency (ESA) is acknowledged for providing the Swarm data and for financially supporting the work described in this paper (ESA/ESTEC contract no. 4000102140/10/NL/JA). The Swarm data used here are the preliminary LP data (data identifier: SW_PREL_EFIx_LP_1B) freely accessible at https://earth.esa.int/web/guest/swarm/data-access. J. Park was partially supported by the "Planetary system research for space exploration" project, the basic research funding from KASI, and the Air Force Research Laboratory, under agreement number FA2386-14-1-4004. G. Malhotra and J. B. H. Baker acknowledge support from the US National Science Foundation under grant AGS-1150879 and ATM-0946900.

The topical editor H. Kil thanks the anonymous referee for help in evaluating this paper.

\section{References}

Benton, C. J. and Mitchell, C. N.: New method for tracking the movement of ionospheric plasma, J. Geophys. Res., 117, A09317, doi:10.1029/2012JA017836, 2012.

Förster, M. and Haaland, S.: Interhemispheric differences in ionospheric convection: Cluster EDI observations revisited, J. Geophys. Res. Space Science, doi:10.1002/2014JA020774, in press, 2015.
Friis-Christensen, E., Lühr, H., Knudsen, D., and Haagmans, R.: Swarm-An Earth Observation Mission investigating Geospace, Adv. Space Res., 41, 210-216, doi:10.1016/j.asr.2006.10.008, 2008.

Goodwin, L. V., Iserhienrhien, B., Miles, D. M., Patra, S., van derMeeren, C., Buchert, S. C., Burchill, J. K., Clausen, L. B. N., Knudsen, D. J., McWilliams, K. A., and Moen, J.: Swarm in situ observations of $\mathrm{F}$ region polar cap patches created by cusp precipitation, Geophys. Res. Lett., 42, 996-1003, doi:10.1002/2014GL062610, 2015.

Hosokawa, K., Shiokawa, K., Otsuka, Y., Nakajima, A., Ogawa, T., and Kelly, J. D.: Estimating drift velocity of polar cap patches with all-sky airglow imager at Resolute Bay, Canada, Geophys. Res. Lett., 33, L15111, doi:10.1029/2006GL026916, 2006.

Lühr, H., Park, J., Gjerloev, J. W., Rauberg, J., Michaelis, I., Merayo, J. M. G., and Brauer, P.: Field-aligned currents' scale analysis performed with the Swarm constellation, Geophys. Res. Lett., 42, 1-8, doi:10.1002/2014GL062453, 2015.

Matsuo, T. and Richmond, A. D.: Effects of high-latitude ionospheric electric field variability on global thermospheric Joule heating and mechanical energy transfer rate, J. Geophys. Res., 113, A07309, doi:10.1029/2007JA012993, 2008.

Nishimura, Y., Lyons, L. R., Zou, Y., Oksavik, K., Moen, J. I., Clausen, L. B., Donovan, E. F., Angelopoulos, V., Shiokawa, K., Ruohoniemi, J. M.,Nishitani, N., McWilliams, K. A., and Lester, M.: Day-night coupling by a localized flow channel visualized by polar cap patch propagation, Geophys. Res. Lett., 41, 37013709, doi:10.1002/2014GL060301, 2014.

Noja, M., Stolle, C., Park, J., and Lühr, H.: Long-term analysis of ionospheric polar patches based on CHAMP TEC data, Radio Sci., 48, 289-301, doi:10.1002/rds.20033, 2013.

Ritter, P., Lühr, H., and Rauberg, J.: Determining field-aligned currents with the Swarm constellation mission, Earth Planets Space, 65, 1285-1294, 2013.

Sojka, J. J., Zhu, L., Crain, D. J., and Schunk, R. W.: Effect of high-latitude ionospheric convection on Sun-aligned polar caps, J. Geophys. Res., 99, 8851-8863, doi:10.1029/93JA02667, 1994.

Spicher, A., Cameron, T., Grono, E. M., Yakymenko, K. N., Buchert, S. C., Clausen, L. B. N., Knudsen, D. J., McWilliams, K. A., and Moen, J. I.: Observation of polar cap patches and calculation of gradient drift instability growth times: A Swarm case study, Geophys. Res. Lett., 42, 201-206, doi:10.1002/2014GL062590, 2015.

Yang, B., Donovan, E., Liang, J., Ruohoniemi, J. M., and Spanswick, E.: Using Patchy Pulsating Aurora to Remote Sense Magnetospheric Convection, Geophys. Res. Lett., doi:10.1002/2015GL064700, in press, 2015.

Zhang, Q.-H., Zhang, B.-C., Lockwood, M., Hu, H.-Q., Moen, J., Ruohoniemi, J. M., Thomas, E. G., Zhang, S.-R., Yang, H.-G, Liu, R.-Y., McWilliams, K. A., and Baker, J. B. H.: Direct Observations of the Evolution of Polar Cap Ionization Patches, Science, 339, 1597-1600, doi:10.1126/science.1231487, 2013.

Zou, Y., Nishimura, Y., Lyons, L. R., Shiokawa, K., Donovan, E. F., Ruohoniemi, J. M., McWilliams, K. A., and Nishitani, N.: Localized polar cap flow enhancement tracing using airglow patches: Statistical properties, IMF dependence, and contribution to polar cap convection, J. Geophys. Res. Space Science, 120, 40644078, doi:10.1002/2014JA020946, 2015. 